\title{
Risk of venous thromboembolism in patients receiving adjuvant chemotherapy with 5-fluorouracil, epirubicin and cyclophosphamide for early breast cancer
}

\author{
Julia HOY, ${ }^{1}$ Teresa NEEMAN, ${ }^{3}$ Robin STUART-HARRIS ${ }^{1,2}$ and Alison DAVIS ${ }^{1,2}$ \\ ${ }^{1}$ The Australian National University Medical School, ${ }^{2}$ Medical Oncology Unit, Canberra Hospital, Woden, ACT, Australia and \\ ${ }^{3}$ Statistical Consulting Unit, The Australian National University, Acton
}

\begin{abstract}
Aim: Previous studies have shown that adjuvant chemotherapy in early breast cancer (EBC) may increase the risk of venous thromboembolism (VTE). Clinical experience suggests the combination of 5-fluorouracil, epirubicin and cyclophosphamide (FEC) may be associated with a higher frequency of VTE than other regimens. This study aims to investigate the use of adjuvant FEC compared with other adjuvant regimens in the development of VTE in patients with EBC.

Methods: A retrospective audit was conducted examining all eligible patients who received adjuvant chemotherapy for EBC in the Australian Capital Territory from 1 January 2005 to 30 June 2007. Data were collected from patients' notes, including risk factors for VTE, tumor pathology, chemotherapy details and incidence of VTE. Comparisons using $\chi^{2}$ tests and independent samples $t$-tests were made between patients who received FEC and those who received another regimen. Multivariate logistic regression was used to investigate prognostic factors for the development of VTE.

Results: A total of 325 patients were included in the study, of whom 176 received FEC and 149 received other adjuvant chemotherapy regimens. The incidence of VTE in patients who received FEC was 47/176 $(27 \%)$, which was significantly higher than for patients who received other regimens $(7 / 149,5 \%, P<0.001)$. FEC was the only significant prognostic factor for the development of VTE (OR 7.9, 95\% CI 3.3-19.2, $P<0.001)$.
\end{abstract}

Conclusion: The use of adjuvant FEC chemotherapy is associated with an increased incidence of VTE in patients with EBC compared with other commonly used chemotherapy regimens.

Key words: adjuvant chemotherapy, early breast cancer, FEC, venous thromboembolism.

\section{INTRODUCTION}

There is a strong association between malignancy and the development of venous thromboembolism (VTE) although the exact mechanism for the association remains unknown. ${ }^{1-3}$ It is believed that tumor cells enhance pro-coagulant pathways such as the coagulation cascade and also inhibit anticoagulant properties. ${ }^{4}$

Correspondence: Dr Alison Davis, Medical Oncology Unit, Canberra Hospital, Woden, ACT 2606, Australia.

Email: alison.davis@act.gov.au

Accepted for publication 16 January 2009.
The development of VTE is a leading cause of death among cancer patients and is also associated with significant morbidity, including local symptoms such as pain and swelling, the risk of pulmonary embolization, the need for long-term anticoagulation and the accompanying risk of bleeding. ${ }^{3,5,6}$

Although breast cancer is associated with a lower incidence of VTE compared with other common malignancies its consequences for morbidity and mortality remain significant. ${ }^{7}$ In patients with breast cancer the presence of metastatic disease is the most significant risk factor for the development of VTE, although the effect of VTE on survival is greater for patients with early stage disease. ${ }^{8}$ 
Studies in patients with early breast cancer (EBC) have demonstrated an additional increase in the risk of VTE associated with the use of chemotherapy and hormonal therapy. ${ }^{9-13}$ Proposed mechanisms for chemotherapy-related thrombosis include the release of pro-coagulants from tumor cells, endothelial damage and stimulation of tissue factor activity in monocytes and macrophages. ${ }^{4,14,15}$

Despite being a known risk factor for the development of VTE in breast cancer patients little is known about which chemotherapy regimens are associated with the greatest risk. A number of studies have looked at the incidence of thrombosis associated with cyclophosphamide, methotrexate and 5-fluorouracil (CMF), a commonly used regimen for $\mathrm{EBC}$ before the introduction of anthracycline-based chemotherapy. ${ }^{10,11,13}$ However, limited studies have investigated the risk of thrombosis with newer regimens. ${ }^{9}, 12$ We suspected an increased incidence of VTE in women having 5-fluorouracil, epirubicin and cyclophosphamide (FEC) compared with other adjuvant chemotherapy regimens used in our clinical practice.

In the current study we aimed to identify the incidence of VTE in a cohort of patients with EBC and specifically investigate the incidence of VTE associated with adjuvant FEC compared with other adjuvant regimens. Additionally, we aimed to examine the possibility of patient variables that may be used as prognostic factors for the development of VTE.

\section{METHODS}

Data were collected from all patients who received adjuvant chemotherapy for the treatment of $\mathrm{EBC}$ in the Australian Capital Territory (ACT) from 1 January 2005 to 30 June 2007 . The study was exempt from ethics approval by the ACT human research ethics committee as it met the guidelines for a clinical audit. Data were obtained from four sites including the Canberra Hospital, the National Capital Private Hospital, Zita Mary Clinic at Calvary Hospital and John James Private Hospital.

Patients who received any treatment with i.v. cyclophosphamide over the specified time period were identified using information obtained from the pharmacy department at each site. As all adjuvant breast cancer regimens used in the ACT during this period included i.v. cyclophosphamide, this captured the group of interest. Patients without breast cancer or with metastatic disease were then excluded by reviewing their records or by checking with the treating physician. The inclusion crite- ria included a diagnosis of EBC, no evidence of metastatic disease at diagnosis and treatment with adjuvant chemotherapy where the first cycle of treatment was commenced between 1 January 2005 and 30 June 2007.

Each record was reviewed and data from those patients who met the inclusion criteria were collected including the patients' characteristics, tumor pathology, chemotherapy regimen and incidence of VTE (superficial or deep), as well as the method of detection where applicable. Patients' data collected at the first cycle of chemotherapy included their age, height, weight, body surface area, concomitant medications, smoking status, menopausal status and previous history of thrombosis. In cases where menopausal status was not indicated in the clinical history, women under the age of 50 years were considered premenopausal and those aged 50 years and above were considered postmenopausal. Their body mass index (BMI) was calculated using a standard equation and used as a comparative measure of weight, scaled to height. The normal range for healthy weight is between 20 and $25 \mathrm{~kg} / \mathrm{m}^{2}{ }^{2}{ }^{16}$

Their recorded tumor pathology included the histological type of breast cancer (ductal, lobular or other), the tumor grade, stage (International Union against Cancer, UICC),${ }^{17}$ hormone receptor status and human epidermal growth factor 2 (Her 2/Erb B2) status. Tumors that were either or both estrogen or progesterone receptor positive were recorded as hormone receptor positive.

The information regarding chemotherapy included the type of regimen, number of cycles received and the route of i.v. administration (by peripheral cannula or by a central venous catheter). For those patients with an identified VTE, the following data were also recorded: the type of VTE, its location, the method of detection and the cycle of chemotherapy. Incidents of thrombosis were classified as superficial, including superficial thrombophlebitis and superficial or deep venous thrombosis, including central line associated thrombosis, deep venous thrombosis (DVT) and pulmonary embolus (PE). Veins in the arm were considered deep if they corresponded to an artery of the same name.

\section{Statistical analysis}

All data were analyzed using SPSS software version 16 (SPSS, Chicago IL, USA). The patients were split into two groups: those who received treatment with FEC, either alone or as part of a sequential regimen of adjuvant chemotherapy such as FEC followed by a taxane, and those who did not receive any FEC. Baseline characteristics were summarized by group, using means 
and standard deviations (SD) for continuous data, and frequency and percentage for categorical data. Differences in baseline characteristics between the two groups were assessed using an independent samples $t$-test for continuous data and a $\chi^{2}$ test for categorical data. An exact $\chi^{2}$ test using a Monte Carlo simulation was used in cases where expected cell counts were less than five. Logistic regression was used to assess the effect of possible prognostic factors for the development of VTE and to assess the association between FEC and the risk of VTE, when adjusting for any identified factors. All significance tests were two-sided and $P$-values less than 0.05 were considered statistically significant.

\section{RESULTS}

In total, 492 patients received treatment with intravenous cyclophosphamide over the specified time period, of which 323 were eligible for inclusion in the study. Three additional patients were included who did not satisfy the initial criteria because the primary tumor was not identified. However, each received adjuvant chemotherapy in concordance with the treatment for EBC as they presented with axillary lymphadenopathy and were assumed to have an occult breast cancer and were therefore deemed suitable. Data were insufficient for one patient, resulting in her removal from the study. Therefore, the final study population consisted of 325 patients from all four sites.

\section{Patient characteristics}

The patient characteristics are detailed in Tables 1 and 2. Most of the patients were postmenopausal $(60 \%)$, although their mean age at the first cycle of chemotherapy was 51.4 years ( \pm SD 10.2). Their mean BMI was slightly above the normal range at $27 \mathrm{~kg} / \mathrm{m}^{2}( \pm \mathrm{SD}$ $5.5)$. Only 18 patients $(5.5 \%)$ had a documented history of previous venous thrombosis ranging from superficial thrombophlebitis to DVT and PE.

The patients' characteristics were well matched between the two groups with no statistically significant differences in continuous or categorical variables except

Table 1 Continuous patient characteristics for the entire cohort (total $n=325$ ) and for each patient group (5-fluorouracil, epirubicin and cyclophosphamide [FEC] $n=176$, non-FEC $n=149$ )

\begin{tabular}{|c|c|c|c|c|c|c|c|c|c|}
\hline & \multicolumn{4}{|c|}{ Total } & \multicolumn{2}{|c|}{ FEC } & \multicolumn{2}{|c|}{ Non-FEC } & \multirow[b]{2}{*}{$P$} \\
\hline & Min & Max & Mean & SD & Mean & SD & Mean & SD & \\
\hline Age (years) & 28 & 76 & 51.4 & 10.2 & 52.6 & 10.0 & 50 & 10.4 & $0.02 *$ \\
\hline Weight (kg) & 43.4 & 142 & 71.7 & 15.1 & 72.7 & 15.6 & 70 & 14.5 & 0.16 \\
\hline Height $(\mathrm{cm})$ & 145 & 181 & 162.5 & 6.3 & 162 & 6.4 & 162.5 & 6.3 & 0.88 \\
\hline BMI $\left(\mathrm{kg} / \mathrm{m}^{2}\right)$ & 17 & 50 & 27 & 5.5 & 27.6 & 5.6 & 26 & 5.4 & 0.14 \\
\hline
\end{tabular}

"Statistically significant difference at the $P<0.05$ level (independent samples 2-tailed t-test). BMI, body mass index;

$\mathrm{SD}$, standard deviation.

Table 2 Categorical patient characteristics for the entire cohort (total $n=325$ ) and for each patient group (5-fluorouracil, epirubicin and cyclophosphamide [FEC] $n=176$, Non-FEC $n=149$ )

\begin{tabular}{|c|c|c|c|c|c|c|c|c|}
\hline & & \multicolumn{2}{|c|}{ Total } & \multicolumn{2}{|c|}{ FEC } & \multicolumn{2}{|c|}{ Non-FEC } & \multirow[b]{2}{*}{$P$} \\
\hline & & $n$ & $\%$ total & $n$ & $\%$ total & $n$ & $\%$ total & \\
\hline \multirow[t]{2}{*}{ Menopause } & Pre & 131 & 40.3 & 67 & 38 & 64 & 43 & \multirow[t]{2}{*}{0.37} \\
\hline & Post & 194 & 59.7 & 109 & 62 & 85 & 57 & \\
\hline \multirow[t]{3}{*}{ Smoker } & Yes & 46 & 14.2 & 23 & 13 & 23 & 15 & \multirow[t]{3}{*}{0.33} \\
\hline & No & 235 & 72.3 & 133 & 76 & 102 & 68 & \\
\hline & Unknown & 44 & 13.2 & 20 & 11 & 24 & 17 & \\
\hline \multirow[t]{3}{*}{ Past history thrombosis } & Yes & 18 & 5.5 & 9 & 5.1 & 9 & 6.0 & \multirow[t]{3}{*}{0.97} \\
\hline & No & 299 & 92 & 163 & 92.6 & 136 & 91.3 & \\
\hline & Unknown & 8 & 2.5 & 4 & 2.3 & 4 & 2.7 & \\
\hline \multirow[t]{2}{*}{ Venous access } & Peripheral & 223 & 68.6 & 116 & 66 & 107 & 71.8 & \multirow[t]{2}{*}{0.25} \\
\hline & Central & 102 & 31.4 & 60 & 34 & 42 & 27.2 & \\
\hline
\end{tabular}


Table 3 Tumor characteristics for the entire cohort (total $n=325$ ) and for each patient group (5-fluorouracil, epirubicin and cyclophosphamide [FEC] $n=176$, Non-FEC $n=149$ )

\begin{tabular}{|c|c|c|c|c|c|c|c|c|}
\hline & & \multicolumn{2}{|c|}{ Total } & \multicolumn{2}{|c|}{ FEC } & \multicolumn{2}{|c|}{ Non-FEC } & \multirow[b]{2}{*}{$P$} \\
\hline & & $n$ & $\%$ total & $n$ & $\%$ total & $n$ & $\%$ total & \\
\hline \multirow[t]{3}{*}{ Type } & Ductal & 283 & 87.1 & 148 & 84.1 & 135 & 90.6 & 0.18 \\
\hline & Lobular & 29 & 8.9 & 20 & 11.4 & 9 & 6.0 & \\
\hline & Other & 13 & 4.0 & 8 & 4.5 & 5 & 3.4 & \\
\hline \multirow[t]{4}{*}{ Grade } & 1 & 23 & 7.1 & 12 & 6.8 & 11 & 7.4 & 0.43 \\
\hline & 2 & 108 & 33.2 & 56 & 31.8 & 52 & 34.9 & \\
\hline & 3 & 192 & 59.1 & 108 & 61.4 & 84 & 56.4 & \\
\hline & Unknown & 2 & 0.6 & 0 & 0 & 2 & 1.3 & \\
\hline \multirow[t]{3}{*}{ Stage $^{\dagger}$} & I & 55 & 16.9 & 23 & 13.1 & 32 & 21.5 & 0.10 \\
\hline & II & 183 & 56.3 & 101 & 57.4 & 82 & 55.0 & \\
\hline & III & 87 & 26.8 & 4 & 29.5 & 35 & 23.5 & \\
\hline \multirow[t]{2}{*}{ Hormone receptor } & Positive & 238 & 73.2 & 143 & 81.2 & 95 & 63.8 & $<0.001^{*}$ \\
\hline & Negative & 87 & 26.8 & 33 & 18.8 & 54 & 36.2 & \\
\hline \multirow[t]{3}{*}{ Her 2 Expression } & Positive & 67 & 20.6 & 33 & 18.8 & 34 & 22.8 & 0.68 \\
\hline & Negative & 252 & 77.5 & 140 & 79.5 & 112 & 75.2 & \\
\hline & Unknown & 6 & 1.8 & 3 & 1.7 & 3 & 2.0 & \\
\hline
\end{tabular}

"Statistically significant difference at the $P<0.05$ level $\left(\chi^{2}\right.$ test). ${ }^{\dagger}$ UICC stage ${ }^{17}$. Stage I, T1N0M0; stage II, T1-3N1M0, T0-2N1M0; stage III, T4N0M0, T3-4N1M0, T0-4N2M0, T0-4N3M0.

for age, as shown in Table 1. On average the FEC group was slightly older with a mean age of $52.6( \pm$ SD 9.9) years compared to $50.0( \pm$ SD 10.4$)$ years for the nonFEC group $(P=0.02)$.

\section{Tumor pathology}

The tumor characteristics are summarized in Table 3. Most of tumors were infiltrating ductal carcinomas of the breast $(283 / 325,87 \%)$ and were high grade (192/ $325,59 \%$ grade 3$)$. Their hormone receptor status was predominantly positive $(73 \%)$ as opposed to Her2 overexpression, which was positive in only $21 \%$ of patients. Overall 83 percent of patients were IUCC stages II or III. The FEC and non-FEC groups were similar with respect to tumor type, grade, stage and Her 2 status. However, in the FEC group 143/176 (81\%) tumors were hormone receptor positive, compared to $95 / 149(64 \%)$ in the non-FEC group $(P<0.001)$.

\section{Chemotherapy}

Data concerning the use of different adjuvant chemotherapy regimens are given in Table 4 . The most commonly used chemotherapy regimens were FEC100 with no sequential chemotherapeutic agents $(95 / 325,29 \%)$ and doxorubicin with cyclophosphamide (AC, 67/325, $21 \%)$. A total of 176 patients $(54 \%)$ received treatment with FEC, either alone or sequentially with other agents, compared to 149 patients $(46 \%)$ in the non-FEC group. In the FEC group 149/176 patients received an epirubicin dose of $100 \mathrm{mg} / \mathrm{m}^{2}$ (FEC100, 85\%) as opposed to 27 patients who received FEC with an epirubicin dose of $75 \mathrm{mg} / \mathrm{m}^{2}$ (FEC75, 15\%).

\section{VTE}

Overall $54(17 \%)$ patients developed some form of VTE while undergoing chemotherapy, 47 of whom were receiving treatment with FEC $(87 \%)$. Of the seven who were not receiving FEC three were receiving treatment with AC, two with epirubicin and cyclophosphamide (EC) and two with docetaxel, doxorubicin and cyclophosphamide (TAC).

The overall incidence of VTE was significantly higher in patients who received FEC than those who received another regimen. In the FEC group, 47/176 (27\%) patients developed a VTE compared to seven out of 149 patients $(5 \%)$ in the non-FEC group $(P<0.001)$. Both superficial and deep VTE were statistically more common in patients receiving FEC when analyzed separately (see Table 5). Although numerically there were more superficial than deep VTE in both groups this difference was not statistically significant $(P=0.52)$.

Thirty-six of the $47(77 \%)$ FEC patients developing a VTE had a peripheral venous access device and $11(23 \%)$ had a central venous access device. In the non-FEC group 
Table 4 Adjuvant chemotherapy regimens (total $n=325$ ) for fluorouracil/epirubicin/cyclophosphamide (FEC) and non-FEC

\begin{tabular}{|c|c|c|c|c|c|}
\hline & \multicolumn{2}{|c|}{ FEC } & & \multicolumn{2}{|c|}{ Non-FEC } \\
\hline & $n$ & $\%$ total & & $n$ & $\%$ total \\
\hline FEC 100 & 95 & 29.2 & $\mathrm{EC}$ & 5 & 1.5 \\
\hline FEC 75 & 23 & 7.1 & $\mathrm{AC}$ & 67 & 20.6 \\
\hline FEC 100 - docetaxel & 31 & 9.5 & $\mathrm{AC} / \mathrm{EC}$ - taxane & 49 & 15.1 \\
\hline FEC 75 - docetaxel & 2 & 0.6 & TAC & 20 & 6.2 \\
\hline FEC 100 - weekly paclitaxel & 16 & 4.9 & TAC - paclitaxel & 1 & 0.3 \\
\hline FEC $100-$ EC & 2 & 0.6 & $\mathrm{CMF}$ & 3 & 0.9 \\
\hline FEC 75 - EC & 2 & 0.6 & Other & 4 & 1.2 \\
\hline Other $^{\dagger}$ & 5 & 1.5 & & & \\
\hline Subtotal & 176 & 54.2 & & 149 & 45.8 \\
\hline
\end{tabular}

${ }^{\dagger}$ Other FEC regimens contained FEC 100 only. AC, doxorubicin/cyclophosphamide; CMF, cyclophosphamide/methotrexate/fluorouracil; EC, epirubicin/cyclophosphamide; FEC 75, epirubicin dose $75 \mathrm{mg} / \mathrm{m}^{2}$; FEC 100, epirubicin dose $100 \mathrm{mg} / \mathrm{m}^{2}$; TAC, docetaxel/doxorubicin/ cyclophosphamide.

Table 5 Incidence and type of venous thromboembolism for the total cohort $(n=325)$ and within each group (FEC $n=176$, non-FEC $n=149$ )

\begin{tabular}{|c|c|c|c|c|c|c|c|c|}
\hline & & \multicolumn{2}{|c|}{ Total } & \multicolumn{2}{|c|}{ FEC } & \multicolumn{2}{|c|}{ Non-FEC } & \multirow[b]{2}{*}{$P$} \\
\hline & & $n$ & $\%$ total & $n$ & $\%$ total & $n$ & $\%$ total & \\
\hline \multirow[t]{4}{*}{ Superficial } & SVT Tx $\operatorname{arm}^{\dagger}$ & 22 & 6.8 & 20 & 11.3 & 2 & 1.3 & \\
\hline & SVT other & 1 & 0.3 & 1 & 0.6 & - & - & \\
\hline & STP Tx arm & 14 & 4.3 & 12 & 6.8 & 2 & 1.3 & \\
\hline & Subtotal & 37 & 11.4 & 33 & 18.7 & 4 & 2.7 & $<0.001^{*}$ \\
\hline \multirow[t]{4}{*}{ Deep } & DVT Tx arm & 10 & 3.1 & 7 & 5.0 & 3 & 2.0 & \\
\hline & DVT other & 3 & 0.9 & 3 & 1.7 & - & - & \\
\hline & $\mathrm{PE}$ & 4 & 1.2 & 4 & 2.3 & - & - & \\
\hline & Subtotal & 17 & 5.2 & 14 & 8.0 & 3 & 2.0 & $0.02 *$ \\
\hline Total & & 54 & 16.6 & 47 & 26.7 & 7 & 4.7 & $<0.001^{*}$ \\
\hline
\end{tabular}

"Statistically significant difference at the $P<0.05$ level $\left(\chi^{2}\right)$. DVT, deep vein thrombosis; FEC, fluorouracil/epirubicin/cyclophosphamide; PE, pulmonary embolus; STP, superficial thrombophlebitis; SVT, superficial venous thrombosis; ${ }^{\dagger} \mathrm{Tx}$ arm, treatment arm.

five of seven patients $(71 \%)$ had a peripheral device and two $(29 \%)$ had a central venous access device. There was no significant difference in the incidence of VTE dependent on the type of venous access device.

Of the patients who received FEC followed by a taxane, all VTE occurred during the FEC portion of their treatment. Most thrombotic events occurred during the first three cycles of chemotherapy (46/54, $85 \%)$, and were predominantly diagnosed using a Doppler ultrasound $(33 / 54,61 \%)$. Thrombotic events occurred in $28 \%(41 / 149)$ patients who received FEC100 compared with $22 \%(6 / 27)$ patients who received a lower dose of epirubicin (FEC75). This difference was not statistically significant.

In addition to the 54 cases of VTE that occurred during chemotherapy, seven patients had documented
VTE that occurred outside the period of treatment with chemotherapy and were not included in the risk factor analysis. Of these, four patients developed portacath-associated DVT one to 12 months after completing adjuvant chemotherapy. Two of the four patients were being treated with trastuzumab (9 and 12 months post-chemotherapy), another with both trastuzumab and tamoxifen (5 months postchemotherapy) and one patient on no other targeted therapy (1 month post-chemotherapy). Two further patients developed DVT, one of which progressed, resulting in a PE. The seventh patient developed a DVT following the insertion of a peripherally inserted central catheter line prior to the administration of chemotherapy. No deaths were recorded as a result of VTE. 


\section{Risk factors}

The use of adjuvant FEC was associated with an increase in the risk of VTE with an OR of 7.9 (95\% CI 3.3-19.2, $P<0.001)$. Other risk factors were assessed as either single variables or using multivariate logistic regression to account for the effect of FEC, although none were statistically significant. These included age, BMI, menopausal status, smoking status, past history of venous thrombosis, tumor pathology, grade and stage, hormone receptor status, Her2 status and type of venous access for administering the chemotherapy.

\section{DISCUSSION}

The incidence of VTE in this study $(17 \%)$ was considerably higher than that identified in previous studies involving patients treated with chemotherapy for EBC $(1.5-10 \%)^{9-13}$ and was comparable to that reported by Goodnough et al. for patients with metastatic breast cancer $(18 \%) .{ }^{18}$ Many of these EBC studies examined DVT only, which may explain the comparatively low incidence of thrombosis, however a study by Levine et al. found that $8.8 \%$ of patients treated with adjuvant chemotherapy for stage II breast cancer developed either superficial or deep VTE. ${ }^{11}$ When considering DVT alone, the incidence of $5 \%$ in the current study lies within the range already documented in the literature for patients treated with chemotherapy for EBC.

It is possible our VTE rate was higher than expected due to the inclusion of superficial thrombophlebitis, which is a diagnosis open to clinical interpretation and is not included in other studies. As this causes notable morbidity to patients in terms of local pain and the limitation of cannula sites for subsequent treatments we believe it should be incorporated into prospective studies to accurately assess incidence rates. It is also possible that our clinical suspicion biased our detection in the FEC group and overestimated the results. An analysis of a separate retrospective data set might help assess whether this was the case.

Previous studies have identified a number of risk factors that increase the likelihood of VTE in patients with breast cancer receiving adjuvant chemotherapy. These include the use of hormonal therapy in conjunction with chemotherapy, postmenopausal status, age greater than 50 years, greater body weight, tumor hormone receptor positivity and a history of mastectomy rather than lumpectomy. ${ }^{9-11,13}$ The slightly older age of patients receiving FEC might have played some role in the increased incidence of VTE in the FEC group, but concomitant hormonal therapy was not given and the type of surgery was not investigated. However, when accounting for the effect of these factors using a logistic regression, the use of FEC was still highly statistically significant in the development of VTE. Similarly, the presence of a central venous catheter had no apparent impact on the development of VTE despite the known risks, and there were no reported cases of arterial thrombosis as described previously. ${ }^{19,20}$

Although at present there are no studies comparing the incidence of VTE with the use of FEC compared to AC or EC, in a study by von Tempelhoff et al the incidence of VTE in patients receiving EC for EBC was $10 \% .^{12}$ In the current study only 10 patients received treatment with EC and two developed VTE (20\%), whereas 114 patients received AC and only three developed VTE (3\%). A small study by Demirkan et al. investigated the effect of FEC and EC on fibrinolytic system markers and the D-dimer levels were significantly higher in the FEC group, although they were still within the normal range and none of the patients developed VTE. ${ }^{21}$

Dose escalation of epirubicin from $50 \mathrm{mg} / \mathrm{m}^{2}$ to $100 \mathrm{mg} / \mathrm{m}^{2}$ in adjuvant FEC has been associated with an increased incidence of acute toxicity including neutropenia, anemia, alopecia, nausea and vomiting. ${ }^{22}$ In the current study FEC100 was associated with a somewhat higher incidence of VTE compared to FEC75, which may suggest a dose effect of epirubicin in the development of VTE. However, the result was not statistically significant. Given that only 27 patients received FEC75 it is difficult to make any unequivocal conclusion about the nature of this dose relationship, although anecdotal evidence suggests that FEC100 is more of a problem.

The incidence of VTE in patients who received treatment with FEC was significantly higher than in patients who received another type of adjuvant chemotherapy including EC. Most thrombotic complications in the FEC group were superficial venous thromboses. Preclinical research has shown that 5-fluorouracil damages the endothelium, particularly in vessels close to the site of administration rather than systemically, and that this damage may be associated with the development of thrombosis. ${ }^{23,24}$ This may, at least in part, explain the higher incidence of superficial thrombosis in patients receiving FEC. However, due to the small number of thrombotic events in the non-FEC group it was difficult to identify any significant correlation.

The use of 5-fluorouracil has also been shown to reduce the activity of protein $\mathrm{C}$ while promoting the activity of fibrinopeptide $\mathrm{A}$, resulting in a pathophysiological picture similar to the activation of coagulation. ${ }^{25}$ Additionally, 5-fluorouracil is known to increase 
platelet aggregation in vitro, which may also enhance coagulation. ${ }^{26}$ Given that FEC was the only adjuvant regimen in the current study that incorporated the use of 5-fluorouracil this raises the possibility that 5fluorouracil may play a causative role in the pathogenesis of FEC-related VTE. CMF, which also contains 5FU, has previously been shown to be associated with increased risk of VTE. ${ }^{10,11,13}$

The current study has shown a significant increase in the incidence of VTE with the use of adjuvant FEC compared with other adjuvant regimens in the treatment of EBC, confirming our clinical impression. There is a need for prospective studies to better quantify the problem as well as investigate the pathogenesis, in particular the potential role of 5-fluorouracil and identify means of prevention, or at least risk minimization.

Current recommendations do not support the use of prophylactic anticoagulants in the outpatient setting for either adjuvant or palliative chemotherapy. ${ }^{27,28}$ However, in other specific clinical situations, such as the use of thalidomide or lenalidomide in combination with chemotherapy and dexamethasone in multiple myelomas, a high incidence of VTE was noted and prophylaxis with low molecular weight heparin is now commonplace. ${ }^{29}$ If it is confirmed that FEC is associated with an excessive risk, then this may need to be considered. In the meantime patient education about the symptoms associated with VTE and increased clinical vigilance is required when treating patients with adjuvant FEC to ensure timely diagnosis and optimal treatment of VTE.

\section{ACKNOWLEDGEMENTS}

The authors would like to thank Ms Katrina Stevanovic, Mrs Lindsay Gatenby, Ms Olivia Barri, Ms Annette Hodges and Mr Stephen Connors for their invaluable assistance in retrieving and organizing medical records. We would also like to thank Drs David Leong and Nicole Gorddard, and Associate Professors Paul Craft and Desmond Yip from Canberra Hospital, ACT, for their help in identifying patients for inclusion in the study.

\section{REFERENCES}

1 Rickles FR, Edwards RL. Activation of blood coagulation in cancer: Touseau's syndrome revisited. Blood 1983; 62: 14-31.

2 Lip GY, Chin BS, Blann AD. Cancer and the prothrombotic state. Lancet Oncol 2002; 3: 27-34.

3 Lee AYY, Levine MN. Venous thromboembolism and cancer: risks and outcomes. Circulation 2003; 107: 1-17.
4 Caine GJ, Stonelake PS, Rea D, Lip GY. Coagulopathic complications in breast cancer. Cancer 2003; 98: 157886.

5 Sorensen HT, Mellemkjaer L, Olsen JH, Baron JA. Prognosis of cancers associated with venous thromboembolism. N Engl J Med 2000; 343: 1846-50.

6 Khorana AA, Francis CW, Culakova E, Kuderer NM, Lyman GH. Thromboembolism is a leading cause of death in cancer patients receiving outpatient chemotherapy. J Thromb Haemost 2007; 5: 632-4.

7 Chew HK, Wun T, Harvey D, Zhou H, White RH. Incidence of venous thromboembolism and its effect on survival among patients with common cancers. Arch Intern Med 2006; 166: 458-64.

8 Chew HK, Wun T, Harvey DJ, Zhou H, White RH. Incidence of venous thromboembolism and the impact on survival in breast cancer patients. J Clin Oncol 2007; 25: 70-6.

9 Clahsen PC, van den Velde CJ, Julien JP, Florias JL, Mignolet FY. Thromboembolic complications after perioperative chemotherapy in women with early breast cancer: a European Organization for Research and Treatment of Cancer Breast Cancer Cooperative Group study. J Clin Oncol 1994; 12: 1266-71.

10 Weiss RB, Tormey DC, Holland JF, Weinbery VE. Venous thrombosis during multimodal treatment of primary breast carcinoma. Cancer Treat Rep 1981; 65: 677-79.

11 Levine MN, Gent M, Hirsh J et al. The thrombogenic effect of anticancer drug therapy in women with stage II breast cancer. N Engl J Med 1988; 318: 404-07.

12 von Tempelhoff GF, Deitrich M, Hommel G, Heilmann L. Blood coagulation during adjuvant epirubicin/cyclophosphamide in patients with primary operable breast cancer. J Clin Oncol 1996; 14: 2560-68.

13 Saphner T, Tormey DC, Gray R. Venous and arterial thrombosis in patients who received adjuvant therapy for breast cancer. J Clin Oncol 1991; 9: 286-94.

14 Doll DC, Ringenberg QS, Jarbro JW. Vascular toxicity associated with antineoplastic agents. Semin Oncol 1992; 19: 580-96.

15 Levine MN. Adjuvant therapy and thrombosis: how to avoid the problem? Breast 2007; 16: S169-74.

16 Eknoyan G. Adolphe Quetelet (1796-1874) - the average man and indices of obesity. Nephrol Dial Transplant 2008; 23: 47-51.

17 Sobin LH, Wittekind C. TNM Classification of Malignant Tumours, 6nd edn. Wiley-Liss, New York 2002.

18 Goodnough LT, Saito H, Manni A, Jones PK, Pearson OH. Increased incidence of thromboembolism in stage IV breast cancer patients treated with a five-drug chemotherapy regimen. A study of 159 patients. Cancer 1984; 54: 1264-8.

19 Kuter DJ. Thrombotic complications of central venous catheters in cancer patients. Oncologist 2004; 9: 20716. 
20 Wall JG, Weiss RB, Norton L et al. Arterial thrombosis associated with adjuvant chemotherapy for breast carcinoma: a Cancer and Leukemia Group B study. Am J Med 1989; 87: 501-04.

21 Demirkan B, Ozcan MA, Glu AA, Yuksel F, Undar B, Alakavuklar M. The effect of anthracycline-based (epirubicin) adjuvant chemotherapy on plasma TAFI and PAI-1 levels in operable breast cancer. Clin Appl. Thromb Haemost 2006; 12: 9-14.

22 Group FAS. Benefit of a high-dose epirubicin regimen in adjuvant chemotherapy for node-positive breast cancer patients with poor prognostic factors: 5-year follow-up. J Clin Oncol 2001; 19: 602-11.

23 Cwikiel M, Zhang B, Eskilsson J, Wieslander JB, Albertsson $\mathrm{M}$. The influence of 5 -fluorouracil on the endothelium in small arteries. An electron microscopic study in rabbits. Scanning Microsc 1995; 9: 561-76.

24 Cwikiel M, Eskilsson J, Wieslander JB, Stjernquist U, Albertsson M. The appearance of endothelium in small arteries after treatment with 5-fluorouracil. An electron microscopic study of late effects in rabbits. Scanning Microsc 1996; 10: 805-18; [Discussion 19].

25 Kuzel T, Esparaz B, Green D, Kies M. Thrombogenicity of intravenous 5-fluorouracil alone or in combination with cisplatin. Cancer 1990; 65: 885-89.

26 Takano S. Effects of antitumour drugs on dog platelet aggregation. Fukushima Med J 1978; 28: 227-33.

27 Lyman GH, Khorana AA, Falanga A et al. American Society of Clinical Oncology guideline: recommendations for venous thromboembolism prophylaxis and treatment in patients with cancer. J Clin Oncol 2007; 25: 5490505.

28 Lee AY. Cancer and venous thromboembolism: prevention, treatment and survival. J Thromb Thrombolysis 2008; 25: 33-6.

29 Klein U, Kosely F, Hillengass J et al. Effective prophylaxis of thromboembolic complications with low molecular weight heparin in relapsed multiple myeloma patients treated with lenalidomide and dexamethasone. Ann Hematol 2009; 88: 67-71. 